\title{
Corrigendum: Cardiac function associated with home ventilator care in Duchenne muscular dystrophy
}

\author{
Sangheun Lee, MD'1, Heeyoung Lee, MD', Lucy Youngmin Eun, MD, PhD'1 Seung-Woong Gang, MD, PhD² \\ ${ }^{1}$ Division of Pediatric Cardiology, Department of Pediatrics, Yonsei University College of Medicine, Seoul, Korea \\ ${ }^{2}$ Department of Rehabilitation Medicine, Gangnam Severance Hospital, Rehabilitation Institute of Neuromuscular Disease, Yonsei University College of Medicine, Seoul, \\ Korea
}

Korean Journal of Pediatrics 2018;61(2):59-63. Published online: February 28, 2018

https://doi.org/10.3345/kjp.2018.61.2.59

In this paper, the fourth author's name and institution were given incorrectly. The correct name should be 'Seong-Woong Kang' instead of 'Seung Woong Gang.' The correct institution should be 'Department of Rehabilitation Medicine, Gangnam Severance Hospital, Rehabilitation Institute of Neuromuscular Disease, Yonsei University College of Medicine, Seoul, Korea' instead of 'Department of Rehabilitation Medicine, Yonsei University College of Medicine, Seoul, Korea.'

Corrected fourth author's name and institution

Sangheun Lee, $\mathrm{MD}^{1}$, Heeyoung Lee, $\mathrm{MD}^{1}$, Lucy Youngmin Eun, $\mathrm{MD}, \mathrm{PhD}^{1}$, Seong-Woong Kang, $\mathrm{MD}, \mathrm{PhD}^{2}$

${ }^{1}$ Division of Pediatric Cardiology, Department of Pediatrics, Yonsei University College of Medicine, Seoul, Korea

${ }^{2}$ Department of Rehabilitation Medicine, Gangnam Severance Hospital, Rehabilitation Institute of Neuromuscular Disease, Yonsei University College of Medicine, Seoul, Korea 